\title{
Protective Effect of SIRT1 Activator on the Knee With Osteoarthritis
}

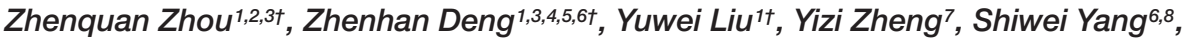 \\ Wei Lu', Deming Xiao ${ }^{3}$ and Weimin Zhu ${ }^{1,3,4,5,6 *}$
}

\begin{abstract}
1 Department of Sports Medicine, The First Affiliated Hospital of Shenzhen University, Shenzhen Second People's Hospital, Shenzhen, China, ${ }^{2}$ Department of Orthopaedics, Shenzhen Hospital of Southern Medical University, Shenzhen, China, ${ }^{3}$ Clinical Medical College, Guangzhou Medical University, Guangzhou, China, ${ }^{4}$ Clinical Medical College, Shenzhen University, Shenzhen, China, ${ }^{5}$ Clinical Medical College, Guangxi University of Chinese Medicine, Nanning, China, ${ }^{6}$ Clinical Medical College, Anhui Medical University, Hefei, China, ’ Department of Thyroid and Breast Surgery, Shenzhen Breast Tumor Research Center for Diagnosis and Treatment, National Standardization Center for Breast Cancer Diagnosis and Treatment, Shenzhen Second People's Hospital, The First Affiliated Hospital of Shenzhen University, Shenzhen, China, ${ }^{8}$ Teaching Office, Shenzhen Second People's Hospital, Shenzhen, China
\end{abstract}

\section{OPEN ACCESS}

Edited by:

Elizabeth S. Fernandes, Pelé Pequeno Príncipe Research Institute, Brazil

Reviewed by: Gábor Pozsgai, University of Pécs, Hungary Marcelo N. Muscará, University of São Paulo, Brazil

*Correspondence: Weimin Zhu

szhzwm@email.szu.edu.cn

${ }^{t}$ These authors have contributed equally to this work

Specialty section:

This article was submitted to Integrative Physiology, a section of the journal Frontiers in Physiology

Received: 31 January 2021 Accepted: 24 March 2021 Published: 13 April 2021

Citation:

Zhou Z, Deng Z, Liu Y, Zheng Y, Yang S, Lu W, Xiao D and Zhu W (2021) Protective Effect of SIRT1

Activator on the Knee With

Osteoarthritis.

Front. Physiol. 12:661852. doi: 10.3389/fphys.2021.661852
Osteoarthritis $(\mathrm{OA})$, one of the most common chronic musculoskeletal disorders, is deemed to be correlated with aging. The SIRT1 activator, resveratrol, acts as a crucial regulator of aging and may have a potential therapeutic effect on OA. Rabbit OA models were established through destabilized medial meniscus surgery. A total of 40 healthy male New Zealand rabbits were divided into five groups: control group (sham operation), OA group, as well as low dose (LD), middle dose (MD), and high dose (HD) resveratroltreated OA groups. 6 weeks after operation, $0.8 \mathrm{ml}$ of normal saline was injected into the knee joints every other day in the control and OA groups, and $0.8 \mathrm{ml}$ of 5,10 , and $15 \mu \mathrm{mol} / \mathrm{L}$ resveratrol was injected into the knee joints every other day in the LD, MD, and HD group, respectively. The rabbits were sacrificed 2 weeks after medication, and the articular cartilage of the knee joint was collected for Micro-CT, histology and Western blot analysis. Obvious articular cartilage lesion and joint space narrowing were detected in the OA group. Compared with the OA group, less osteoarthritic changes were observed in the MD and HD groups. The MD and HD groups had significantly lower bone volume fraction, trabecular number and Mankin scores than the LD and OA groups $(p<0.05)$. No significant difference was found between the OA and LD groups ( $p>0.05)$. The expressions of SIRT1 and p53 detected by western blot were consistent with the aforementioned findings. Therefore, resveratrol can activate the SIRT1 gene to play a protective role in the OA process by inhibiting chondrocyte apoptosis, trabecular bone number increasing of the subchondral bone, as well as elevation of bone density. It demonstrated the importance of SIRT1 in maintaining articular cartilage health and provided a promising therapeutic intervention in the treatment of OA.

Keywords: osteoarthritis, resveratrol, SIRT1, p53, cartilage, Micro-CT

\footnotetext{
Abbreviations: 3D, Three-dimentional; AGEs, Advanced glycation end-products; BCA, Bicinchoninic acid; BV/TV, Bone volume/tissue volume ratio; DAB, Diaminobenzidine; DMM, Destabilized medial meniscus; EDTA, Ethylene diamine tetraacetic acid; FOXOs, Forkhead box O; H\&E, Hematoxylin and eosin; HD, High dose; IGF, Insulin-like growth factor; KL, Kellgren-Lawrence; LD, Low dose; MD, Middle dose; MMPs, Metalloproteinases; mTOR, Mammalian target of rapamycin; NF-кB, Nuclear factor-kappaB; OA, Osteoarthritis; PBS, Phosphate buffer saline; PI3K, Phosphatidylinositol 3-kinase; ROS, Reactive oxygen species; SD, Standard deviation; SIRT 1, Sirtuin 1; Tb.N, Trabecular number; TNF- $\alpha$, Tumor necrosis factor- $\alpha$; Tb.Th, Trabecular thickness.
} 


\section{INTRODUCTION}

Osteoarthritis (OA) is one of the most common chronic musculoskeletal disorders (Glyn-Jones et al., 2015; Deng et al., 2018). There are multiple treatments in clinical practice, but none of them can inhibit the pathological processes effectively. Arthroplasty is an effective treatment for end-stage OA; however, in view of the serious complications of surgery and limited lifespan of prostheses, researchers are pursuing treatments for early OA and measures for disease prevention (Glyn-Jones et al., 2015). The epidemiology of OA is complex and multifactorial, and has not yet been fully understood, so how to treat $\mathrm{OA}$ is still a great challenge worldwide. Among various risk factors, agerelated changes in the phenotype of articular chondrocytes are key factors in the development and progression of OA (Rahmati et al., 2017). The mechanism of aging in relation to OA, which is still unclear, could provide targets for therapy in OA treatments.

Sirtuins are members of the class III histone deacetylase family, which mainly regulate diverse cellular activities in aging and aging-related diseases (Deng et al., 2019b). The silent information regulator 2 type 1 (also known as sirtuin 1 [SIRT 1]) is the most popular sirtuin homologs and regulates many vital signaling pathways such as DNA repair and apoptosis, myogenic and adipogenic differentiation, mitochondrial biogenesis, and glucose and insulin homeostasis (Hubbard and Sinclair, 2014). Resveratrol (Res, 3, 5, and 4'-trihydroxy-trans-stilbene), a non-flavonoid polyphenol compound, can activate the SIRT1 gene (Deng et al., 2019b). Studies have examined its antiinflammatory, antioxidant, anti-free radical, anticancer effects as well as the function of improving micro-circulation (Ota et al., 2006; Moussa et al., 2017; Xia et al., 2017). Although evidence has shown that the SIRT1 gene plays an important role in chondrocyte apoptosis, but the specific mechanism is still unclear (Takayama et al., 2009). A study on the other cell line indicated that the equilibrium state of nuclear factor-kappaB (NF- $\mathrm{B}$ ) and p53 regulated by the SIRT1 gene had a significant impact on cell survival (Day et al., 2001). A previous study also revealed that the forkhead box O (FOXOs) gene regulated chondrocyte apoptosis by modulating the expressions of apoptosis-related factors (Goldring and Goldring, 2010). The interaction between SIRT1 and FOXOs improves cell survival (Shane Anderson and Loeser, 2010), and provides a basis for investigating the role of SIRT1 in regulating OA progression.

As a type of gene closely related to cell differentiation, aging and apoptosis, the role that the SIRT1 gene plays in the occurrence and development of OA is still unclarified. In this study, we supposed that the SIRT1 gene participated in chondrocyte aging and apoptosis by modulating the expressions of NF- $\mathrm{KB}$ and $\mathrm{p} 53$. Meanwhile, the therapeutic effect of resveratrol in rabbit OA models was explored.

\section{MATERIALS AND METHODS}

\section{Ethics Statement}

The animal experiment was carried out in accordance with relevant guidelines and regulations, and was approved by the
Medical Ethics Committee of the First Affiliated Hospital of Shenzhen University, Shenzhen Second People’s Hospital.

\section{Animals and Grouping}

A total of 403 -month old male mature New Zealand white rabbits $(1.8 \pm 0.17 \mathrm{~kg})$ provided by Guangdong Medical Laboratory Animal Center were used in this study. Each of the rabbits was housed in an individual cage under the following conditions: $21 \pm 1^{\circ} \mathrm{C} ; 12 \mathrm{~h} / 12 \mathrm{~h}$ light/dark cycle; $40-60 \%$ relative humidity; and fed with water, rabbit chow, and green leaves.

All the rabbits were randomly divided into five groups (eight in each group): control group (sham operation), OA group (OA induction with normal saline injection), LD group (low dose resveratrol, OA induction with $5 \mu \mathrm{mol} / \mathrm{L}$ of resveratrol injection), $\mathrm{MD}$ group (moderate dose resveratrol, $\mathrm{OA}$ induction with $10 \mu \mathrm{mol} / \mathrm{L}$ of resveratrol injection), and $\mathrm{HD}$ group (high dose resveratrol, OA induction with $15 \mu \mathrm{mol} / \mathrm{L}$ of resveratrol injection).

\section{Destabilized Medial Meniscus - Induced OA Model}

All surgeries were performed under isoflurane anesthesia, and the rabbits were given postsurgical buprenorphine $(0.05 \mathrm{mg} / \mathrm{kg}$ subcutaneously) as analgesia.

According to the reference, destabilized medial meniscus (DMM) surgery was performed randomly on one side of the lower leg to create OA models (Deng et al., 2019a). Briefly, the rabbit was fixed on the operation table with the right leg in $90^{\circ}$ flexion. The joint capsule was opened with an incision just medial to the patellar tendon, and the medial meniscotibial ligament was sectioned. Then, meniscus was removed to eventually achieve the OA model. Sterility requirements were followed during operation. Joints were wrapped by iodine gauze and fixed with elastic bandage. Normal feeding was performed after operation for 2 weeks. In the first 3 days postoperatively, 800,000 units of penicillin (Shijiazhuang Pharmaceutical Group, China) were intramuscularly injected on a daily basis to prevent infection.

Six weeks after operation, $0.8 \mathrm{ml}$ of normal saline was injected into the articular cavity of the surgery knee every other day in the control group, and $0.8 \mathrm{ml}$ of resveratrol at the concentration of 5,10 , and $15 \mu \mathrm{mol} / \mathrm{L}$ (Shenzhen Xinhailing Biotechnology Co., Ltd.) was injected into the knee joints every other day in the LD, $\mathrm{MD}$, and $\mathrm{HD}$ group, respectively.

\section{Sample Preparation and Cell Culture}

Rabbits were sacrificed 8 weeks after operation. Biopsies of the cartilage and bone were acquired from the tibia plateau, including the loading zone and the margin zone as far as possible (Deng et al., 2017). Specimens were examined by Micro-CT. Articular cartilage was removed from the articular surface by sterile scalpel and was transferred to $4^{\circ} \mathrm{C}$ sterile DMEM (Hyclone, United States). After washing with phosphate buffer saline, it was then moved to a $150 \mathrm{ml}$ flask containing $0.25 \%$ trypsinethylene diamine tetraacetic acid (EDTA, Gibico, United States). Samples were stirred and incubated for $1 \mathrm{~h}$ at $37^{\circ} \mathrm{C}$ and $95 \%$ humidity. Liquid was discarded and $0.02 \%$ collagenase II with 
$5 \%$ calf serum was added. Subsequently, samples were stirred and incubated overnight, and were filtered and centrifuged on the next day. Precipitates of chondrocytes were incubated with unfiltered cartilage. Culture medium was replaced every 3 days. Subculture was performed when chondrocytes covered $80 \%$ of the surface of culture medium.

\section{X-Ray Observation}

Full leg X-rays of rabbits were captured using a Direct Radiography image system (SHIMADZU, Japan, $400 \mathrm{~mA}$; Radiology Department, Shenzhen Second People's Hospital). Kellgren-Lawrence (KL) classification score (0-4) was used to evaluate disease severity. A higher score indicates a higher level of OA severity.

\section{Micro-CT Analysis}

The proximal tibia was scanned with a Scanco Viva CT 40 (Scanco Medical, Wangen-Brüttisellen, Switzerland) at $15-\mathrm{mm}$ resolution 8 weeks after surgery. The X-ray energy was $100 \mathrm{~mA}$ and $50 \mathrm{kVp}$. The slice thickness was $15 \mathrm{~mm}$. After obtaining 2-dimensional image slices, the field of interest was uniformly delineated, and three-dimensional (3D) reconstructions were performed with gauss $=0.8, \mathrm{~s}=1$, and threshold $=163$ throughout the analysis. The bone volume/tissue volume ratio (BV/TV), trabecular number (Tb.N), trabecular thickness (Tb.Th), and the changes of $3 \mathrm{D}$ structure were analyzed through MicroCT scanning.

\section{Histology}

Dissected joints were fixed overnight at $4^{\circ} \mathrm{C}$ in $4 \%$ paraformaldehyde, decalcified for 3 weeks in $0.5 \mathrm{M}$ EDTA $\mathrm{pH}=7.5$, embedded in paraffin, and then sectioned at $5 \mu \mathrm{m}$ thickness in the sagittal plane. The sections were stained with hematoxylin and eosin (H\&E; Deng et al., 2019a). The morphology of the articular cartilage was further evaluated using the Mankin scoring system. A higher score indicates a higher level of severity of cartilage degeneration.

\section{Western Blot}

The second generation of chondrocytes cultured was used to test the signal pathway involved in resveratrol treatment. Chondrocytes were lysed with the RIPA buffer to extract the whole proteins. The total protein content of the extracted sample was quantified with a bicinchoninic acid protein assay. Protein samples $(50 \mu \mathrm{g})$ were separated on $10 \%$ polyacrylamide gel and then transferred to polyvinylidenefluoride membranes. After blocked with 5\% Skim milk/BSA, the membranes were incubated with primary antibodies including: GAPDH (1:1000, \#5174, Cell Signaling Technology, Danvers, MA, United States), SIRT1 (\#p475, 1:1000, Cell Signaling Technology, Danvers, MA, United States), and p53 (1:1000, \#2527, Cell Signaling Technology, Danvers, MA, United States) overnight. After washing, the membranes were then incubated with secondary antibody (1:5000, \#7074; Cell Signaling Technology, Danvers, MA, United States) for $2 \mathrm{~h}$. Finally, the protein was exposed with diaminobenzidine. All experiments were repeated for three times.

\section{Statistical Analysis}

The values were expressed as means \pm standard deviation (SD). All statistical analyses were performed using the SPSS 16.0 software (Chicago, IL, United States). One-way ANOVA followed by LSD's post hoc tests was used to determine the differences among groups. Student's $t$ test was used to compare between two groups. $P$ value $<0.05$ was considered statistically significant.

\section{RESULTS}

\section{X-Ray Observation}

In the control group, no significant change of the joint space was found, and smooth and clear outline of the articular surface was observed both in femoral condyle and tibial plateau. In the OA group, narrowed medial space of knee joint and rough surface of tibial plateau was observed. For the resveratrol treatment groups with different doses, the medial space of knee joint gradually narrowed relative to the control group as the dose decreased, but was wider than that of the OA group. The articular surface of femoral condyle and tibial plateau in the $\mathrm{LD}, \mathrm{MD}$, and $\mathrm{HD}$ groups appeared to be smoother compared to the OA group (Figure 1A).

The OA, LD, MD, and HD groups had higher KL classification scores than the control group $(P<0.05)$. The KL classification scores in the MD and HD groups were significantly lower than those in the OA and LD groups $(P<0.05)$, while no significant difference was found between the $\mathrm{MD}$ and $\mathrm{HD}$ groups, and between the OA and LD groups $(P>0.05$, Figure 1B).

\section{Micro-CT Analysis}

Figure $2 \mathrm{~A}$ presents the $3 \mathrm{D}$ images of proximal tibial. The OA, $\mathrm{LD}, \mathrm{MD}$, and $\mathrm{HD}$ groups had higher $\mathrm{BV} / \mathrm{TV}$ values than the control group $(P<0.05)$. The BV/TV values in the MD and $\mathrm{HD}$ groups were lower than those in the control, OA and LD groups $(P<0.05)$, while no significant difference was found between the $\mathrm{MD}$ and HD groups, and between the control and LD groups $(P>0.05$, Figure 2B).

The Tb.N value showed a consistent trend with BV/TV. The OA, LD, MD, and HD groups had lower Tb.N values than the control group $(P<0.05)$. The Tb.N values in the MD and HD groups were lower than those in the control, OA and LD groups $(P<0.05)$, while no significant difference was found between the $\mathrm{MD}$ and HD groups, and between the control and LD groups $(P>0.05$, Figure 2C).

No significant difference in the $\mathrm{Tb}$.Th value was detected among all the groups ( $p>0.05$, Figure 2D).

\section{Histological Analysis}

H\&E staining showed that chondrocytes were orderly arranged in the control group, with the shallow layer, transitional layer, radiation layer, and calcified layer presented from top to bottom in columnar array. Chondrocyte clusters were not observed and the tidemark was intact. In the OA group, the cartilage surface was rough, cracks were seen, and the chondrocyte number was decreased and disorderly arranged and clustered. The morphology of the cartilage was gradually improved with the increase of resveratrol dose (Figure 3A). 


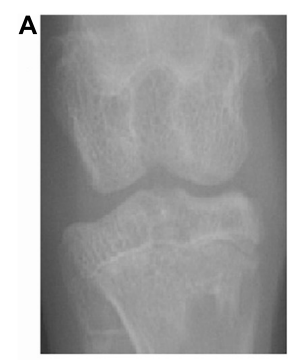

Control

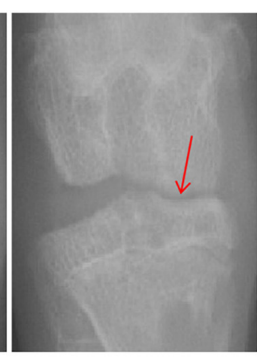

OA

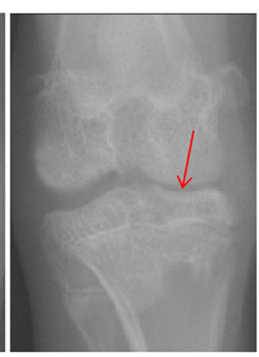

LD

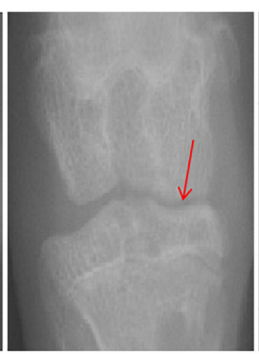

MD

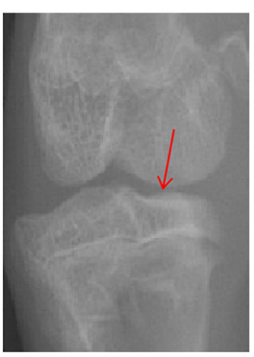

HD

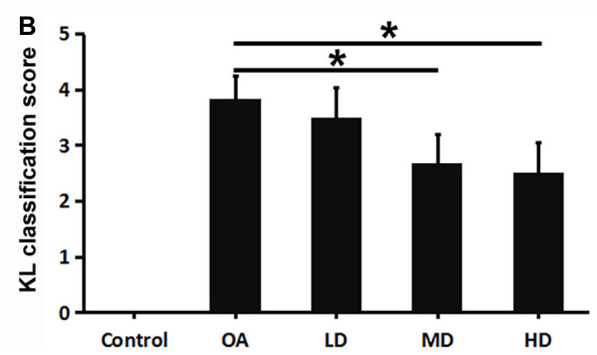

FIGURE 1 |X-ray of the knee in each group at 8 weeks after operation. Arrows showed the joint space narrowing and articular cartilage lesion of the knee joint. (A) $\mathrm{X}$-ray of the knee in each group. (B) $\mathrm{KL}$ classification score of the knee in each group. ${ }^{\star} P<0.05$.
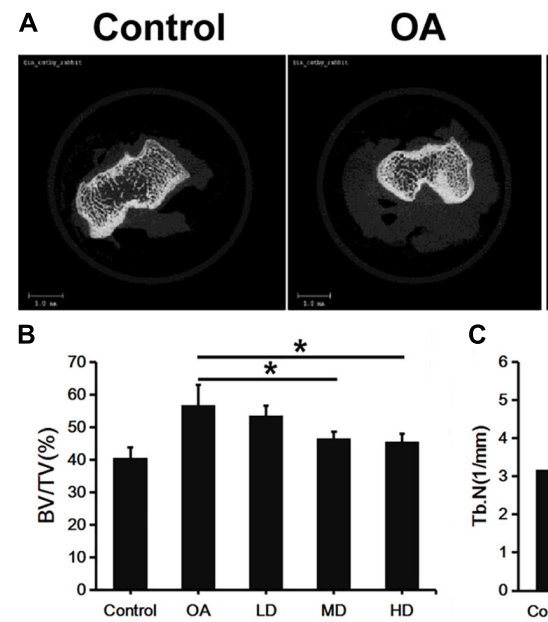

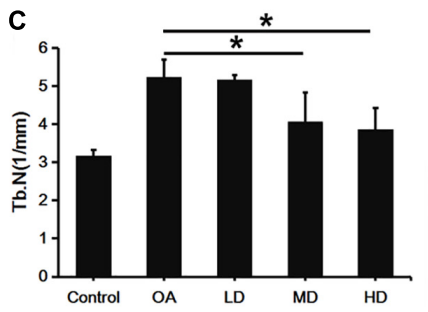

MD

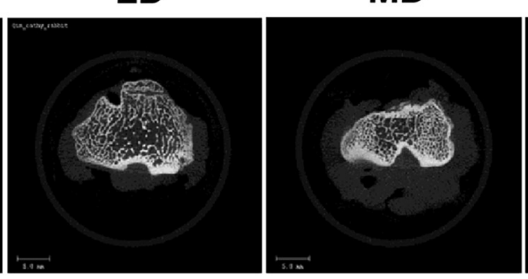

D

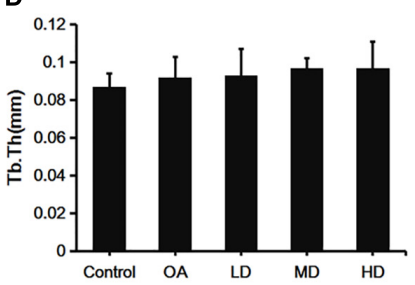

FIGURE 2 | Micro-CT analysis of proximal tibia of difference groups at 8 weeks after surgery. (A) Representative Micro-CT 3D images showed bone microarchitecture of proximal tibia of different groups. Scale bar $=5 \mathrm{~mm}$. (B-D) Quantification of BV/TV, Tb.N, and Tb.Th of different groups. * $P<0.05$.

Mankin score for OA evaluation was adopted in this study. A higher score indicates greater cartilage lesion. As the dose of resveratrol increased, The resveratrol treatment groups presented gradually-decreasing scores compared to the control and OA groups. The $\mathrm{MD}$ and $\mathrm{HD}$ groups showed significantly lower Mankin scores than the $\mathrm{LD}$ and $\mathrm{OA}$ groups $(P<0.05)$. No difference was found between the MD and HD groups, and between the LD and OA groups $(P>0.05$, Figure 3B).

\section{Western Blot Analysis}

The Western blot assay showed that the expression of SIRT1 in the control group was higher than that in other groups
$(P<0.05)$, and the SIRT1 expression in the MD and HD groups was higher than that in the OA and LD groups $(P<0.05)$. The expression of p53 in the OA group was higher than that in other groups $(P<0.05)$, and $\mathrm{p} 53$ expression in the control group was lower than LD group $(P<0.05)$, higher than MD group $(P<0.05)$, and no difference when compared with MD group $(P>0.05$, Figure 4).

\section{DISCUSSION}

In this study, it was found that the SIRT1 activator, resveratrol, inhibited the process of articular cartilage lesion and provided 


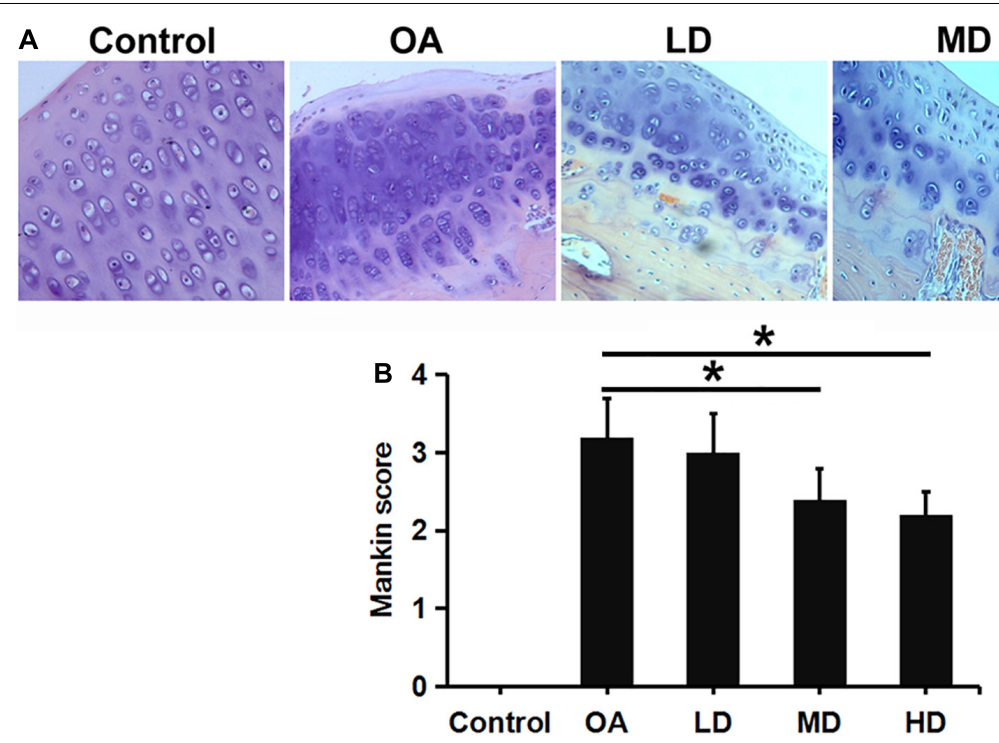

FIGURE 3 | H\&E staining and Mankin score evaluation of articular cartilage of the knee in different groups. (A) H\&E staining showed articular cartilage of the knee in different groups (200X). (B) Mankin score evaluation of different groups. ${ }^{*} P<0.05$.
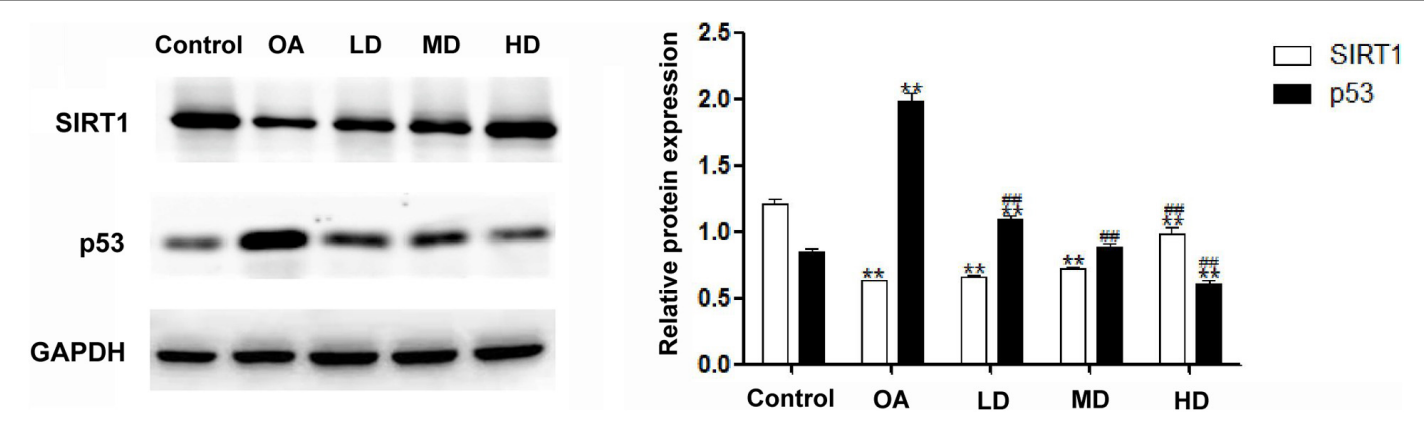

FIGURE 4 | Expressions of SIRT1 and p53 detected by Western blot. ${ }^{\star \star} P<0.05$, compared with the control group; ${ }^{\# \# ~} P<0.05$, compared with OA group.

protection against $\mathrm{OA}$. We revealed a signaling model in cartilage linking the p53 signal pathway with the SIRT1 activator, resveratrol.

The loss of cartilage in subchondral bone is a typical pathological change and feature in OA. In the later stage of $\mathrm{OA}$, the pathological changes of subchondral bone include eburnation, bone cyst, and osteophyte (Chen et al., 2020). We compared BV/TV, Tb.N, and Tb.Th between different groups through Micro-CT scanning. Compared with the LD and OA groups, the HD group exhibited a stronger inhibiting effect on the increase of Tb.N and density, thus retarding the formation of eburnation, bone cyst and osteophyte. Among the various factors, aging of cartilage is an important one contributing to the development of OA. It also exerts a synergism effect along with other OA risk factors such as inflammation, oxidative stress, and changes in the extracellular matrix (Loeser et al., 2016). The reduction of muscle and accumulation of fat will change the joint loading and lead to adipokine and cytokine production and systemic inflammation, which will give impetus to OA (Greene and Loeser, 2015). The excessive reactive oxygen species is correlated with age associated oxidative stress and will result in aging diseases via cellular damage (Jones, 2015). The accumulation of advanced glycation endproducts, which will lead to non-enzymatic collagen crosslinking, can change the mechanical properties of the extracellular matrix of cartilage, thus resulting in cartilage degeneration (Verzijl et al., 2002).

It was suggested that resveratrol, a polyphenol found in grapes, could act as an activator of SIRT1 to protect against oxidative damage, inflammation, and cancer (Lee et al., 2019). In our study, resveratrol exhibited the ability to modulate Tb.N and density. Observation was performed under optical microscope after morphology staining. In the control group, chondrocytes appeared to be normal and orderly arranged, with the shallow layer, transitional layer, radiation layer, and calcified layer presented in columnar array. Chondrocyte clusters were unseen, and the tidal line was intact. The changes in chondrocytes differed among the experimental groups, which accorded with pathological changes in OA. The Mankins score also supported the protective effect of resveratrol with regard to cartilage lesion. 
Resveratrol can regulate multiple molecular targets to increase DNA stability and hinder the process of aging-related diseases (Deng et al., 2019b). It also showed the positive effects of calorie restriction and lifespan extension (Deng et al., 2019b). Meanwhile, resveratrol can inhibit reticulum stress (ER stress) and prevent cell death via autophagy pathways (Lee et al., 2019).

The results of the expressions of SIRT1 and p53 detected by Western blot indicated that resveratrol at different doses could alleviate pathological changes in the $\mathrm{OA}$ model. In the $\mathrm{MD}$ and $\mathrm{HD}$ groups, resveratrol showed a better protective effect than in the LD group, implying that the effect of resveratrol may be concentration dependent. It was further confirmed that resveratrol played an important role in chondrocyte apoptosis by activating SIRT1. Previous studies had confirmed that the expression of SIRT1 decreased in seriously degenerated cartilage (Fujita et al., 2011). Our results showed the same trend: the control group had the highest while the OA group had the lowest SIRT1 expression, and the SIRT1 expression increased with the increase of resveratrol dose. The catabolic, oxidative stress and the pro-inflammatory factors reduced the SIRT1 expression and activity in articular chondrocytes (Deng et al., 2019b). SIRT1 has been suggested to play a vital role in several OA processes. The increased level of SIRT1 is correlated with up-regulated collagen II and aggrecan expression (Oppenheimer et al., 2014). SIRT1 is essential in chondrogenic differentiation of mesenchymal stem cells via NF-кB inhibition and SOX9 activation (Buhrmann et al., 2014). The opposing effects of SIRT1 with matrix metalloproteinases enzymes and tumor necrosis factor- $\alpha$ on cartilage showed the anti-catabolic and anti-inflammatory effects of SIRT1 (Deng et al., 2019b). The anti-oxidative stress effects of SIRT1 were also confirmed through its upregulation after oxidant insult and its decreasing under melatonin treatment in chondrocytes (Lim et al., 2012). The p53 protein is one of the first deacetylated non-histone proteins that are proved to interact with SIRT1. Under cellular stress, p53 is induced by phosphorylation and acetylation and is significantly up-regulated (Tang et al., 2008). Thus, the deacetylation by SIRT1 modulates the function of p53. In this study, along with the alleviation of OA severity, SIRT1 was increased. Consequently, p53 was dramatically decreased in the groups treated with resveratrol, indicating reduction of apoptosis in OA tissue.

Animal models of $\mathrm{OA}$ are used worldwide to address the disease mechanism and to investigate prevention methods and treatments. As rabbits share a similar structure of knee with human, they can be utilized to help us discover the pathological processes, features, and metabolism of OA. Thus, the choice of DMM-OA model in our study is feasible. This method is worldwide recognized and adopted. A successful animal model is the key to the present study.

In our study, it was confirmed that the SIRT1/p53 signal pathway played an important role in aging and apoptosis in chondrocytes (Deng et al., 2019b). A previous study also reported the anti-apoptosis and autophagy effect of SIRT1 by activating the insulin-like growth factor, the phosphatidylinositol 3-kinase signal, and the mammalian target of rapamycin pathway (Gagarina et al., 2010). Above all, our study provided evidence on the protective effect of resveratrol in articular cartilage via the SIRT1/p53 signal pathway. In view of the low bioavailability of resveratrol, further investigation is needed to improve its bioavailability and therapeutic effects in clinical trials.

\section{CONCLUSION}

Resveratrol can activate the SIRT1 gene to play a protective role in the OA process by inhibiting chondrocyte apoptosis, trabecular bone number increasing of the subchondral bone, and elevation of bone density. It demonstrated the importance of SIRT1 in maintaining articular cartilage health and provided a promising therapeutic intervention in the treatment of OA.

\section{DATA AVAILABILITY STATEMENT}

The original contributions presented in the study are included in the article/supplementary material, further inquiries can be directed to the corresponding author/s.

\section{ETHICS STATEMENT}

The animal study was reviewed and approved by Medical Ethics Committee of The First Affiliated Hospital of Shenzhen University, Shenzhen Second People's Hospital.

\section{AUTHOR CONTRIBUTIONS}

DX and WZ designed and directed the study. ZZ, ZD, and YL performed the experiments. YZ and ZD analyzed the data. SY and WL collected the data. ZD and YL prepared the manuscript. $\mathrm{ZD}$ and $\mathrm{WZ}$ were responsible for funding acquisition. All authors contributed to the article and approved the submitted version.

\section{FUNDING}

This study was supported by the National Natural Science Foundation of China (Nos. 81902303 to ZD and 81902682 to YZ), Guangdong Basic and Applied Basic Research Foundation (No. 2020A151501048 to ZD), Shenzhen Science and Technology Project (RCBS20200714114856299 and JCYJ20190806164216661 to ZD and GJHZ20180416164801042 and JCYJ20180305124912336 to WZ), Science and Technology Commission of Shanghai Municipality (Shanghai Sailing Program; No. 19YF1408900 to YZ), Clinical Research Project of Shezhen Second People's Hospital (No. 20203357028 to ZD and No. 20203357007 to WZ), and Shenzhen Double Chain Project for Innovation and Development Industry supported by Bureau of Industry and Information Technology of Shenzhen (201806081524201510 to WZ). 


\section{REFERENCES}

Buhrmann, C., Busch, F., Shayan, P., and Shakibaei, M. (2014). Sirtuin-1 (SIRT1) is required for promoting chondrogenic differentiation of mesenchymal stem cells. J. Biol. Chem. 289, 22048-22062. doi: 10.1074/jbc.m114.568790

Chen, Y., Jiang, W., Yong, H., He, M., Yang, Y., Deng, Z., et al. (2020). Macrophages in osteoarthritis: pathophysiology and therapeutics. Am. J. Transl. Res. 12, 261-268.

Day, J. S., Ding, M., van der Linden, J. C., Hvid, I., Sumner, D. R., and Weinans, H. (2001). A decreased subchondral trabecular bone tissue elastic modulus is associated with pre-arthritic cartilage damage. J. Orthop. Res. 19, 914-918. doi: 10.1016/s0736-0266(01)00012-2

Deng, Z. H., Li, Y. S., Gao, X., Lei, G. H., and Huard, J. (2018). Bone morphogenetic proteins for articular cartilage regeneration. Osteoarthritis Cartilage 26, $1153-$ 1161. doi: 10.1016/j.joca.2018.03.007

Deng, Z., Gao, X., Sun, X., Amra, S., Lu, A., Cui, Y., et al. (2019a). Characterization of articular cartilage homeostasis and the mechanism of superior cartilage regeneration of MRL/MpJ mice. FASEB J. 33, 8809-8821. doi: 10.1096/fj. 201802132rr

Deng, Z., Li, Y., Lin, Z., Zhu, Y., and Zhao, R. (2017). The biomechanical and histological effects of posterior cruciate ligament rupture on the medial tibial plateau. J. Orthopaedic Surg. Res. 12:48.

Deng, Z., Li, Y., Liu, H., Xiao, S., Li, L., Tian, J., et al. (2019b). The role of sirtuin 1 and its activator, resveratrol in osteoarthritis. Biosci. Rep. 39:BSR2019 0189.

Fujita, N., Matsushita, T., Ishida, K., Kubo, S., Matsumoto, T., Takayama, K., et al. (2011). Potential involvement of SIRT1 in the pathogenesis of osteoarthritis through the modulation of chondrocyte gene expressions. J. Orthop. 29, 511515. doi: 10.1002/jor.21284

Gagarina, V., Gabay, O., Dvir-Ginzberg, M., Lee, E. J., Brady, J. K., Quon, M. J., et al. (2010). SirT1 enhances survival of human osteoarthritic chondrocytes by repressing protein tyrosine phosphatase $1 \mathrm{~B}$ and activating the insulin-like growth factor receptor pathway. Arthritis Rheumatism 62, 1383-1392. doi: 10.1002/art.27369

Glyn-Jones, S., Palmer, A. J., Agricola, R., Price, A. J., Vincent, T. L., Weinans, H., et al. (2015). Osteoarthritis. Lancet (Lond. Engl.) 386, 376-387.

Goldring, M. B., and Goldring, S. R. (2010). Articular cartilage and subchondral bone in the pathogenesis of osteoarthritis. Ann. N. Y. Acad. Sci. 1192, 230-237. doi: 10.1111/j.1749-6632.2009.05240.x

Greene, M. A., and Loeser, R. F. (2015). Aging-related inflammation in osteoarthritis. Osteoarthritis Cartilage 23, 1966-1971. doi: 10.1016/j.joca. 2015. 01.008

Hubbard, B. P., and Sinclair, D. A. (2014). Small molecule SIRT1 activators for the treatment of aging and age-related diseases. Trends Pharmacol. Sci. 35, 146-154. doi: 10.1016/j.tips.2013.12.004

Jones, D. P. (2015). Redox theory of aging. Redox Biol. 5, 71-79. doi: 10.1016/j. redox.2015.03.004

Lee, J., Hong, S. W., Kwon, H., Park, S. E., Rhee, E. J., Park, C. Y., et al. (2019). Resveratrol, an activator of SIRT1, improves ER stress by increasing clusterin expression in HepG2 cells. Cell Stress Chaperones 24, 825-833. doi: 10.1007/ s12192-019-01012-z
Lim, H. D., Kim, Y. S., Ko, S. H., Yoon, I. J., Cho, S. G., Chun, Y. H., et al. (2012). Cytoprotective and anti-inflammatory effects of melatonin in hydrogen peroxide-stimulated $\mathrm{CHON}-001$ human chondrocyte cell line and rabbit model of osteoarthritis via the SIRT1 pathway. J. Pineal Res. 53, 225-237. doi: 10.1111/ j.1600-079x.2012.00991.x

Loeser, R. F., Collins, J. A., and Diekman, B. O. (2016). Ageing and the pathogenesis of osteoarthritis. Nat. Rev. Rheumatol. 12, 412-420. doi: 10.1038/nrrheum. 2016.65

Moussa, C., Hebron, M., Huang, X., Ahn, J., Rissman, R. A., Aisen, P. S., et al. (2017). Resveratrol regulates neuro-inflammation and induces adaptive immunity in Alzheimer's disease. J. Neuroinflammation 14:1.

Oppenheimer, H., Kumar, A., Meir, H., Schwartz, I., Zini, A., Haze, A., et al. (2014). Set7/9 impacts COL2A1 expression through binding and repression of SirT1 histone deacetylation. J. Bone Miner. Res. 29, 348-360. doi: 10.1002/jbmr.2052

Ota, H., Tokunaga, E., Chang, K., Hikasa, M., Iijima, K., Eto, M., et al. (2006). Sirt1 inhibitor, sirtinol, induces senescence-like growth arrest with attenuated RasMAPK signaling in human cancer cells. Oncogene 25, 176-185. doi: 10.1038/sj. onc. 1209049

Rahmati, M., Nalesso, G., Mobasheri, A., and Mozafari, M. (2017). Aging and osteoarthritis: central role of the extracellular matrix. Ageing Res. Rev. 40, 20-30. doi: 10.1016/j.arr.2017.07.004

Shane Anderson, A., and Loeser, R. F. (2010). Why is osteoarthritis an age-related disease? Best Pract. Res. Clin. Rheumatol. 24, 15-26. doi: 10.1016/j.berh.2009. 08.006

Takayama, K., Ishida, K., Matsushita, T., Fujita, N., Hayashi, S., Sasaki, K., et al. (2009). SIRT1 regulation of apoptosis of human chondrocytes. Arthritis Rheumatism 60, 2731-2740. doi: 10.1002/art.24864

Tang, Y., Zhao, W., Chen, Y., Zhao, Y., and Gu, W. (2008). Acetylation is indispensable for p53 activation. Cell 133, 612-626. doi: 10.1016/j.cell.2008.03. 025

Verzijl, N., DeGroot, J., Ben, Z. C., Brau-Benjamin, O., Maroudas, A., Bank, R. A., et al. (2002). Crosslinking by advanced glycation end products increases the stiffness of the collagen network in human articular cartilage: a possible mechanism through which age is a risk factor for osteoarthritis. Arthritis Rheumatism 46, 114-123. doi: 10.1002/1529-0131(200201)46:1<114::aid$\operatorname{art10025>3.0.c0;2-p~}$

Xia, N., Daiber, A., Förstermann, U., and Li, H. (2017). Antioxidant effects of resveratrol in the cardiovascular system. Br. J. Pharmacol. 174, 1633-1646. doi: $10.1111 /$ bph. 13492

Conflict of Interest: The authors declare that the research was conducted in the absence of any commercial or financial relationships that could be construed as a potential conflict of interest.

Copyright (C) 2021 Zhou, Deng, Liu, Zheng, Yang, Lu, Xiao and Zhu. This is an open-access article distributed under the terms of the Creative Commons Attribution License (CC BY). The use, distribution or reproduction in other forums is permitted, provided the original author(s) and the copyright owner(s) are credited and that the original publication in this journal is cited, in accordance with accepted academic practice. No use, distribution or reproduction is permitted which does not comply with these terms. 